\title{
La mirada de estudiantes de la Universidad Nacional hacia el docente y la docente: sus características y clima de aula
}

\section{National university`s students perspective of teachers and college education: their characteristic and classroom environment}

\author{
Zulay Pereira Pérez ${ }^{1}$ \\ División de Educación Básica del \\ Centro de Investigación y Docencia en Educación (CIDE) \\ Universidad Nacional \\ Heredia, Costa Rica
}

Recibido 22 de marzo de $2010 \bullet$ Aceptado 24 de junio de 2010

Resumen. El artículo presenta los resultados de una investigación acerca de las características de docentes de la Universidad y el clima de aula, desde la mirada estudiantil. Mediante un diseño de método mixto, se acerca a la caracterización docente con la participación del estudiantado de las carreras en Pedagogía. Los resultados destacan la importancia de los rasgos correspondientes a características de personalidad y psicopedagógicas que otorgan las estudiantes, en contraposición a los planteamientos teóricos sobre el tema, que destacan los rasgos de características profesionales.

Palabras clave. Universidad, docencia, estudiantes, docentes, clima de aula, características personales, profesionales, psicopedagógicas, relaciones de poder en el aula.

Abstract. This article presents the results of a research project in which the characteristics of university teachers and classroom environment were studied from the students' perspectives. The study was based on a mixed design, this one attempts to develop a profile of the teachers, with the participation of students being trained in pedagogy majors. The results indicate that the students underline the importance of personality and psico-pedagogical characteristics in contrast with the theoretical findings, which point more towards professional characteristics.

Key words. University, teaching, faculty, classroom environment, personal characteristics, professional characteristics, students, psychopedagogical characteristics, classroom interactions of power.

\footnotetext{
Doctora en Educación, de la Universidad Estatal a Distancia, Costa Rica. Catedrática de la Universidad Nacional, Costa Rica. Máster y Licenciada en Psicología, de la Universidad de Costa Rica. Adscrita al Colegio Profesional de Psicólogos de Costa Rica. Ex coordinadora del Programa de Investigación en Epistemología Genética y Educación del IIMEC, actualmente el Instituto de Investigación en Educación (INIE) de la Universidad de Costa Rica (UCR). Ex Directora y docente de la División de Educación Básica del Centro de Investigación y Docencia en Educación de la Universidad Nacional, Costa Rica. Investigadora del Centro de Investigación y Docencia en Educación de la Universidad Nacional, Costa Rica. Tiene publicaciones en el campo de la Psicología y la Pedagogía. Correo electrónico: zpereira@una.ac.cr
} 


\section{Introducción}

Para la comprensión de la temática propuesta, es necesario señalar que los planteamiento de estudios en el contexto universitario, tanto en el ámbito nacional, como en el internacional (Francis (2006); Campos (2003); Prieto (2007); Camacho y Mendías (s. f); Palomero y Fernández (1999), entre otros), refieren al impacto, sea positivo o negativo, que tienen las dinámicas interactivas en el clima de la clase. También se señala que las características de docentes, su perspectiva epistemológica y las estrategias de enseñanza que utilizan, así como las relaciones que establecen con sus alumnos, tienen una clara repercusión en el clima de aula, con el consecuente impacto en el desarrollo del estudiantado.

A partir de esta perspectiva, se valoran los procesos de enseñanza y aprendizaje con una visión humana, interesada por conocer, desde la óptica de estudiantes universitarios: cuáles son las características básicas que podrían identificar a docentes que desde su ámbito laboral promueven procesos de desarrollo, no solo referidos al ámbito cognitivo, sino también, al desarrollo del estudiante como ser humano integral.

Desde cualesquiera de las ópticas señaladas, es innegable que las acciones del docente y la docente en el aula, llevan a que se promueva o no, un clima democrático o autoritario, que impacta directamente los procesos de enseñanza y aprendizaje.

De manera más sistemática, a partir del año 2000, se encuentran, en el ámbito universitario, planteamientos referidos al perfil docente, más allá de su campo de especialidad y destrezas de enseñanza, dándole un lugar a las emociones y a los sentimientos (Caputo (2007); Allidière (2004); Mata (2004); Hernández (2005), entre otros). Estos enfocan, de manera prioritaria, las capacidades necesarias del docente para responder, apropiadamente, a los cambios sufridos por el mundo en el campo tecnológico, las comunicaciones y la respuesta requerida por los procesos de globalización vividos en el planeta.

Es interesante señalar que dichos estudios se ubican en el contexto universitario y, como se ha señalado anteriormente, destacan aspectos propios del personal docente en su ámbito tanto profesional, como personal y psicopedagógico, pues antes de esa fecha, la priorización de investigaciones se encuentra en los niveles de primaria y secundaria.

Campo (citado por Francis, 2006) menciona que gran parte de los esfuerzos por definir propuestas de perfiles para el personal docente cuyo desempeño ocurre en el nivel universitario, se encuentran en artículos de reflexión y ensayos, pero que son pocos los aportes surgidos de resultados de investigación.

Por lo señalado previamente, se planteó como parte del Trabajo Final de Graduación del Programa de Doctorado en Educación, de la Universidad Estatal a Distancia, el tema de investigación "La mirada de estudiantes de la Universidad Nacional hacia el docente y la docente: sus características y clima de aula" (Pereira, 2010), el cual se propuso abordar la caracterización de los docentes y las docentes universitarias y el clima de aula, desde la óptica del estudiantado universitario.

Los resultados de dicha investigación se constituyeron en el insumo para el presente artículo,

y se espera que sean de utilidad para fortalecer los procesos de enseñanza y aprendizaje dentro del contexto universitario.

Dicha investigación ofrece una mirada desde el estudiantado, el cual, en primera instancia, es quien enfrenta las consecuencias de dinámicas interactivas dentro del aula, las que pueden tener características favorecedoras o entorpecedoras del desarrollo y del aprendizaje.

El planteamiento acerca del personal docente universitario, hecho por Campo (citado por Francis, 2006), señala: 
El docente como persona ve reflejada su condición humana en sus relaciones, mismas que explicitan los valores con que comulga y que se asocian con las creencias y teorías implícitas que marcan las rutas de conversación, trato y aceptación hacia otros. La coherencia entre el discurso del deber ser como persona y su praxis como docente es un requisito tanto deseable como indispensable porque el docente señala lo que espera que aprendan los estudiantes y modela valores y calidades comportamentales que también son aprehendidas por los estudiantes. Los rasgos dentro de esta dimensión se pueden categorizar de acuerdo con el ámbito de relación personal. (p. 12)

El tema de las dinámicas interactivas en el contexto universitario es importante, en especial si se toma en cuenta que la mayoría de los estudios sobre esta temática han sido realizados en los niveles iniciales de la formación educativa (primaria y secundaria). En un mundo cambiante, donde la comunicación y la tecnología marcan una pauta importante, la función docente, también, ha de evolucionar y responder a las necesidades de contextos en continua transformación.

Algunos de los trabajos analizados en el campo universitario acerca de la temática en cuestión (Prieto, 2007; Ortiz y Mariño, 2005; Palomero y Fernández, 1999, entre otros) están referidos a la incorporación de factores afectivos como parte del proceso de enseñanza y aprendizaje. Mencionan también la urgencia de que la universidad responda a las necesidades humanas y de formación, como parte del perfil docente en donde se valora, no sólo la formación en el campo de la especialidad, sino también las capacidades para comunicarse y establecer adecuadas relaciones interpersonales con sus alumnos y alumnas y promoverlas entre ellos y ellas.

En esta perspectiva, se valoran los procesos de enseñanza y aprendizaje desde una visión humana, que se interesa por conocer, desde la mirada de estudiantes universitarios y universitarias, cuáles son las características que podrían identificar a docentes, considerados como personas que desde su ámbito laboral, promueven procesos de desenvolvimiento, no solo referidos al aspecto cognitivo, sino también al desarrollo del estudiante como ser humano integral.

Si se parte de la idea de que las interacciones en el aula promueven o desvalorizan las dinámicas de enseñanza y aprendizaje, es entonces válido suponer que para la existencia de ambientes positivos, que susciten la motivación y el aprendizaje en contextos universitarios, han de existir también docentes que, con sus características personales, profesionales y psicopedagógicas, impulsen el aprendizaje significativo, en un clima propicio para éste.

Contar con una caracterización del personal docente, desde la visión del estudiantado, puede constituirse en un aporte para la búsqueda de dinámicas de aula que garanticen un clima adecuado para las interacciones positivas y apoyaría la promoción de una entrega de la docencia impregnada de humanidad, en donde se respete la diversidad. La caracterización docente como elemento de un posible perfil, desde la mirada estudiantil, garantizaría la búsqueda de personal idóneo para desempeñarse en las aulas universitarias, generador de procesos de enseñanza y aprendizaje democráticos que permitan la formación de personas saludables y felices. Aspectos que podrían también verse reflejados en la labor de la División de Educación Básica que, permanentemente, busca el mejoramiento, no solo a nivel de planes y programas de estudio, sino también en la formación de su personal docente.

De igual manera, es apreciable una investigación como la señalada, si se considera que en carreras que ofrecen formación en el campo educativo, la relación docente-alumno/alumna cobra especial relevancia, ya que el cuerpo docente se constituye en modelo de las dinámicas que luego el estudiantado, podrá reproducir en sus propias prácticas educativas. 
La experiencia personal, desarrollada en el campo de las relaciones interpersonales, se constituye en un valor agregado para incursionar en un campo cuyo análisis se considera valioso en el contexto educativo y, más específicamente, en el ámbito universitario.

La investigación que origina este artículo se desarrolló en la Universidad Nacional, en el 2009 y participaron estudiantes de licenciatura de las carreras de Preescolar, I y II Ciclos y Educación Especial, de la División de Educación Básica.

Dentro del contexto planteado, se incorporaron como problemas, los siguientes:

1. ¿Cuáles son las características del personal docente universitario, desde la perspectiva de los estudiantes y las estudiantes, a partir del análisis de las dinámicas interactivas que se establecen en el salón de clase?

2. ¿Cómo es el clima de aula universitaria, si el personal docente cuenta con las características que sugiere el estudiantado?

\section{Contexto de la investigación}

La educación es un elemento destacable en los proyectos sociales, políticos y culturales de las naciones, ésta suele responder a una visión de ser humano y de mundo que la sociedad desea preservar y fortalecer. Mediante ella, los pueblos transmiten valores, ideas, conocimientos y creencias, pero también mediante ella, pueden promoverse, desde las aulas, cambios en las cosmovisiones que de una u otra manera, pueden incluso llegar a producir transformaciones sociales importantes.

La educación como valor social ha de constituirse en el medio para promover la formación, no sólo desde la perspectiva cognoscitiva, sino también desde la humanización del ser, para que sea capaz de insertarse en una sociedad que día a día se modifica y la cual requiere de profesionales capaces de incorporarse de manera eficaz en el mundo laboral. Las tendencias actuales en cuanto a formación ciudadana enfatizan la necesidad de que la emoción y el sentimiento se constituyan, también, en elementos determinantes en los procesos formativos, ya que los mismos son inherentes al ser humano.

Cabe destacar que la visión que se tenga en cuanto al porqué, el cómo y el para qué de la educación, determina de manera directa las propuestas curriculares de las instituciones formadoras y el estilo en que se relacionan todos los elementos constitutivos que inciden en los procesos de enseñanza-aprendizaje, dentro de los cuales pueden mencionarse los siguientes: aspectos cognoscitivos, emotivos, concepto de aula, condiciones físicas y estructurales del aula, interacciones docente/ alumno, alumno/alumno, contenidos, estrategias metodológicas, función de la evaluación, tipos de evaluación, clima de aula, disciplina, uso del tiempo y espacio, entre otros posibles de destacar.

Existe una clara vinculación entre las propuestas curriculares, los paradigmas en los cuales se sustentan y las dinámicas de aula que de la aplicación de unos u otros se deriva. Por ello es importante retomar la concepción paradigmática de algunas propuestas curriculares y establecer, desde ellas, las dinámicas de aula que es viable suponer que se generarán a partir de la práctica educativa.

Así, paradigmas controladores -como el tecnológico- generarían, posiblemente, un clima de aula controlador, reproductivo y, la mayoría de las veces, represivo.

En una visión más tradicional, cuando se asume un enfoque de educación como transmisora de conocimientos y valores sociales, se enfatiza en el contenido y en la priorización de estrategias de 
trabajo que llevan al uso de la clase magistral, el texto y la pizarra como recursos didácticos. Dentro de esta visión técnica del currículo, la labor del docente y la docente está claramente priorizada y su función radica en transmitir los conocimientos socialmente valorados, y utilizar las estrategias metodológicas como medios para garantizar el aprendizaje y la concordancia del contenido con los objetivos propuestos.

En correspondencia con esa visión, se espera una dinámica de aula en donde prevalezca la función docente, la institución otorgue un valor y un poder explícito al docente, la disciplina cumpla el cargo de hacer funcional la tarea del docente y el papel del estudiante sea más pasivo que activo. Las interacciones docentes con el alumnado se circunscriban a una relación de respeto/temor, acatamiento de reglas, en las cuales la interacción entre alumnos y alumnas esté claramente negada como paralela al proceso de enseñanza-aprendizaje, aunque podrían existir excepciones en función de las características docentes, pero en términos generales lo descrito bien podría ser lo que caracterice las dinámicas de ese tipo de aula.

En una perspectiva constructivista, se esperaría un balance entre los contenidos, las emociones, las interacciones, es decir, la intervención equilibrada de los distintos elementos señalados en el párrafo precedente, como copartícipes de los procesos de enseñanza-aprendizaje. Es tan importante el papel del docente, como persona, profesional y pedagogo, como lo es la del alumno y alumna dentro de su subjetividad y como ser social, en donde los conocimientos anteriores, cultura, experiencias y contexto lo han ido definiendo como persona.

Es el concepto de aprendizaje como proceso activo y dinámico -donde tanto docente como estudiante se encuentran en situación de aprendizaje-, lo que en última instancia lleva al planteamiento de una visión curricular que parte del respeto al otro, del conocer que la motivación es un elemento imprescindible en el proceso de aprendizaje y en el que, también, se valora el contenido, las habilidades, las actitudes como formadoras de conocimiento, valores y creencias.

Desde una visión de aprendizaje significativo, pueden crearse condiciones de aula que susciten de manera positiva los procesos de adquisición de conocimientos y de desarrollo integral, que permitan no solo acceder al conocimiento, sino también, generar comportamientos de respeto, autonomía, solidaridad, donde la incorporación en sociedad sea saludable, pues se requiere de equidad y oportunidad para todos y dejar abierta la opción y el deseo permanente hacia el aprendizaje.

Es importante destacar que la Universidad, como institución educativa, responde a un modelo de sociedad que la hace parte de ella, que le deposita las expectativas de la formación profesional que requiere un mundo en constante transformación como el actual. El modelo educativo de la Universidad condiciona, a su vez, el diseño que adoptan las facultades y dentro de ella la percepción que se tenga acerca de los procesos educativos y formativos.

Es claramente esperable que las dinámicas interactivas desarrolladas en las aulas universitarias constituyan fiel reflejo de los conceptos pedagógicos que en ellas se prioricen, de manera que recopilar las características docentes, desde la perspectiva del estudiantado universitario, resulta un reto interesante para acercarse al conocimiento de las percepciones estudiantiles y del concepto del cuerpo docente como generador de aprendizajes en el ámbito universitario.

La Universidad Nacional dentro de su estructura cuenta con el Centro de Investigación y Docencia en Educación (CIDE), que trabaja a partir de un objeto de estudio común, como lo es la educación. A este se adscriben un Instituto y varias Unidades Académicas, dentro de ellas, la División de Educación Básica, en la cual se desarrolló la investigación durante el año 2009. 


\section{Metodología utilizada}

La investigación realizada retomó el planteamiento de su diseño, enmarcándolo en un Diseño de Método Mixto, compartiendo el criterio que expresan Hernández, Fernández y Baptista (2004), acerca de los diseños mixtos, en el sentido de que:

(...) representa el más alto grado de integración o combinación entre los enfoques cualitativo y cuantitativo. Ambos se entremezclan o combinan en todo el proceso de investigación, o, al menos, en la mayoría de sus etapas (...) agrega complejidad al diseño de estudio; pero contempla todas las ventajas de cada uno de los enfoques. (p. 21)

En concordancia con las clasificaciones sobre diseños de método o modelo mixtos, planteados en la bibliografía, la presente investigación, respondió a los siguientes criterios:

- Se asumió la complementariedad metodológica, con un diseño de método mixto, con status dominante y de orden secuencial cuan $\longrightarrow$ CUAL (Johnson y Onwuegbuzie, 2004; Di Silvestre, 2008).

- En cuanto al tipo de combinaciones, es un diseño TIPO VI, es decir, un diseño mixto de investigación exploratoria con datos cuantitativos y análisis cualitativo (Rocco, Bliss, Gallagher y Pérez, 2003; Salgado, 2007).

- En cuanto al tipo de estrategia seguida, la investigación planteada responde a una estrategia exploratoria, donde a nivel de secuencia es cuantitativa-CUALITATIVA con integración en la interpretación y cuyo énfasis es explicar e interpretar relaciones (Creswell, 2003).

En el sentido expuesto, la investigación realizada se basó en un diseño de método mixto y, aunque recurrió a métodos tanto cuantitativos (fase I) como cualitativos (fase II), se destaca un énfasis en el paradigma naturalista, con un enfoque cualitativo y el recurso del método fenomenológico, que buscó una posible caracterización del docente y la docente, dentro de las dinámicas interactivas en el aula universitaria, desde la mirada estudiantil.

Cabe recordar que Taylor y Bogdan conciben la investigación cualitativa como “(...) aquella que produce datos descriptivos: las propias palabras de las personas, habladas o escritas y la conducta observable" (Citados por Rodríguez, Gómez y Gil (1996), p. 33).

La investigación otorgó un lugar predominante al enfoque cualitativo, dada la necesidad de profundizar en las dinámicas interactivas (docentes y alumnos /alumnas y alumnos y alumnas entre sí), en el clima de aula y en un posible perfil del docente y la docente universitaria a partir de la perspectiva estudiantil.

En el sentido señalado, se incorporaron tres macro categorías de análisis, a saber:

A. Dinámicas interactivas profesor/profesora - alumno/alumna y dinámicas interactivas alumnos-alumnas entre sí

B. Características del docente y la docente universitaria

C. Clima de aula

\section{Fase I: población y muestra}

La población estuvo constituida por estudiantes de las carreras de Licenciatura en Pedagogía, de los énfasis de Preescolar, I y II Ciclos y Licenciatura en Educación Especial con énfasis en Integración, de la División de Educación Básica de la Universidad Nacional. 
La fase I de la investigación tuvo como fuentes de información, la participación de estudiantes del nivel de licenciatura de las carreras de preescolar, I y II Ciclos y Educación Especial.

La selección de participantes se hizo mediante muestreo aleatorio simple, para completar una participación de la siguiente manera: Pre escolar 10 estudiantes, I y II Ciclos 10 estudiantes y Educación Especial, 10 estudiantes.

La fase I conllevó la utilización del método descriptivo, se empleó un cuestionario para recopilar los rasgos del personal docente universitario desde la perspectiva estudiantil.

El instrumento comprendió, básicamente, aspectos referidos a la vivencia del estudiantado en su proceso de formación reflejados en: los rasgos o características reales del personal docente que los forma; las características ideales acerca del personal docente universitario; la perspectiva estudiantil sobre el clima de aula; el tipo de interacción con el personal docente universitario y las interacciones entre estudiantes.

El cuestionario se estructuró mediante reactivos de respuesta abierta y, en casos específicos, se solicitó jerarquizar características, lo cual permitió, posteriormente, identificar los rasgos de los y las docentes, desde la perspectiva estudiantil.

El instrumento fue validado mediante aplicación piloto y por criterio de jueces, manejándose un índice de congruencia de 8.

\section{Fase II: escogencia de participantes}

Una vez establecidas las tendencias que emanaron de los datos aportados por las estudiantes universitarias (Fase I) acerca de las dinámicas interactivas y la caracterización del personal docente, se procedió a solicitar la participación estudiantil para la segunda fase.

Participaron diez estudiantes en total, con representación de los distintos énfasis de carrera. En el conversatorio se contó con la participación voluntaria de tres estudiantes. La escogencia de las participantes fue por conveniencia, con criterios de accesibilidad y disponibilidad de recursos y mediando el recurso de la invitación libre, según Morse (Citado por Valles, 1999).

La Fase II, por su naturaleza introspectiva, asumió el método fenomenológico; buscó la comprensión de características del docente y la docente, y el clima de aula, desde la perspectiva estudiantil. Se nutrió de los datos aportados por una prueba de Oraciones Incompletas y un Conversatorio con las estudiantes.

El método y la técnica utilizados en esta fase permitieron valorar las dinámicas interactivas entre docentes universitarios y estudiantes, así como entre alumnos y alumnas entre sí y el clima de aula. Ambos fueron revisados en cuanto a forma, contenido y su relación con las categorías de análisis, mediante el criterio de jueces y prueba piloto.

La aplicación de los instrumentos y técnicas estuvo a cargo de la investigadora, al igual que la reducción y análisis de los datos. Se recurrió a la triangulación por teoría, informantes y fuentes de recolección de datos. La presentación de datos fue integrada en concordancia con el diseño de método mixto utilizado (Dellinger y Leech, 2007; Driesnnack, Sousa y Costa, 2007).

\section{Resultados obtenidos}

Cabe destacar que los datos aportados por el cuestionario, en la primera fase de la investigación, correspondieron prioritariamente a las dinámicas interactivas vivenciadas por las 
participantes durante los cursos de su carrera. Aspectos que en la fase II fueron profundizados mediante el conversatorio y las oraciones incompletas.

En el sentido señalado, los resultados indicaron con un excelente porcentaje, que para el $100 \%$ de las estudiantes participantes, independientemente del énfasis de carrera, las experiencias vividas en las aulas universitarias durante los cursos que han llevado, fueron calificadas como positivas, argumentando entre las razones aspectos referidos a su propia motivación y esfuerzo como estudiantes y a factores propios de las características personales y profesionales de sus docentes en los cursos.

De igual manera, señalaron que las interacciones con los docentes y las docentes universitarias y el clima de aula fueron buenos, cuando los cursos estuvieron en manos de "buenos y buenas docentes"; lo contrario ocurrió cuando los cursos estuvieron a cargo de "malos y malas docentes". Esta connotación de buenos o malos docentes, se utilizó como recurso para enmarcar el vocabulario que comúnmente es utilizado por el estudiantado al referirse al personal docente y buscar así, aproximarse a sus planteamientos de manera más clara.

Los resultados encontrados coinciden plenamente con los planteamientos teóricos acerca de la temática (Laborda y Essomba, 2000; Pereira, 2008a-2008-b; Gallardo, 2007; Polanco, 2005; Hernández, 2005, entre otros).

Ante la solicitud de caracterizar a los buenos y buenas docentes universitarias, las estudiantes expresaron opiniones que se resumen en la siguiente Figura.

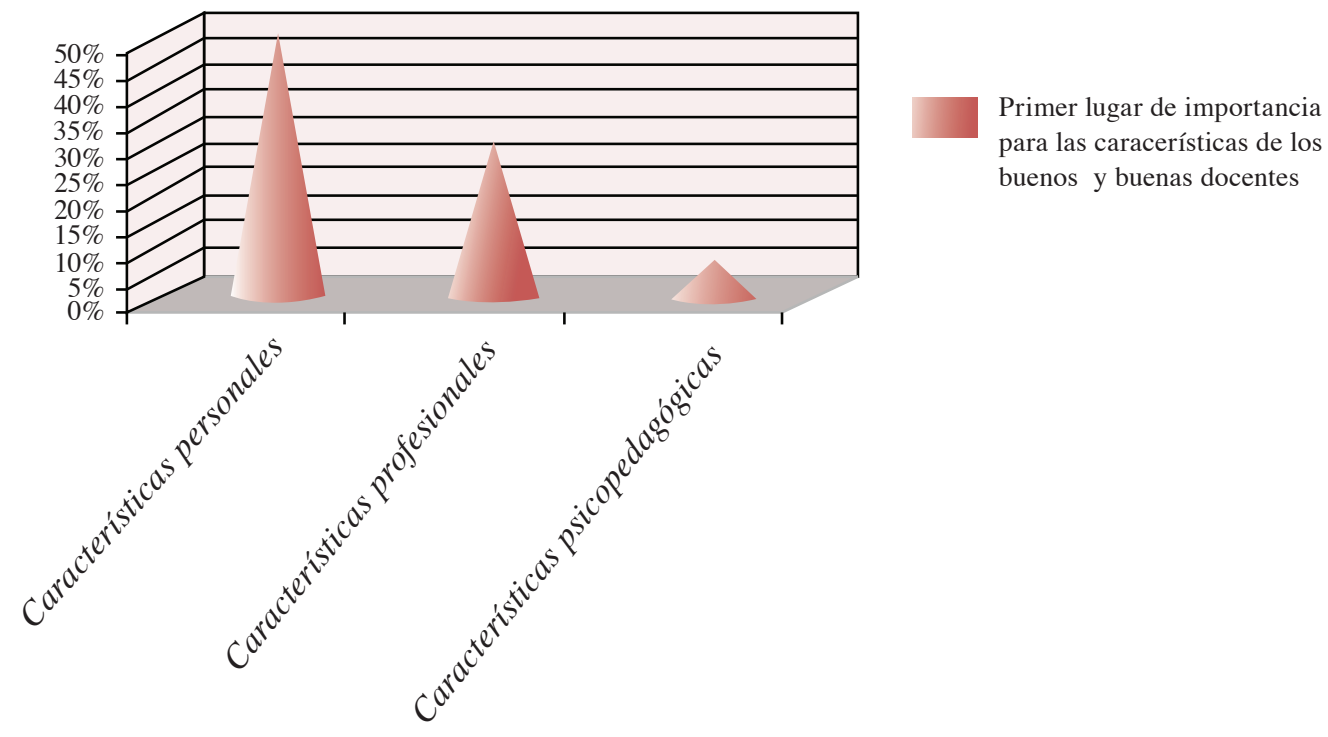

Figura 1. Primer lugar de importancia para las características de un buen y una buena docente universitaria, según opinión de estudiantes de la UNA, 2009.

Como bien se nota en la Figura 1, las estudiantes se refirieron, en primer lugar, con un muy buen porcentaje (50\% de respuestas), a rasgos correspondientes a las características personales del docente y la docente, sin dejar por ello de referirse también a las características profesionales y psicopedagógicas.

En el sentido expuesto, existen claras diferencias entre los datos encontrados y lo que se refiere en la investigación de Mata (2004), dado que los hallazgos en dicho estudio mostraban la prevalencia de la visión profesional y psicopedagógica sobre la personal. Y en el Figura 1, se evidencia, justamente, lo contrario a lo reportado. 
Es interesante destacar que cuando las estudiantes se abocaron a mencionar las características de los buenos y las buenas profesoras universitarias, señalaron lo siguiente:

Tabla 1

Respuestas de las estudiantes de la UNA, ante la frase: Un buen profesor y una buena profesora universitaria se caracteriza por..., en la prueba de oraciones incompletas, 2009

\begin{tabular}{l} 
1. "ser dinámico y empático". \\
\hline 2. "saber muy bien sobre lo que habla (conocer su campo), pero sobre todo, saber transmitirlo y darse a entender". \\
3. "ser flexible y dinámico". \\
4. "saber escuchar a sus estudiantes e ir construyendo el curso según necesidades e intereses de todos". \\
\hline 5. "motivar a los alumnos a aprender". \\
\hline 6. "ser responsable y flexible". \\
\hline 7. "su amor por lo que hace". \\
\hline 8. "ser apasionado con lo que hace y amante de su labor". \\
\hline 9. "su desempeño y amor a su trabajo". \\
10. "llevar al contexto del aula la realidad del exterior y no sólo la teoría".
\end{tabular}

$\mathrm{Al}$ referirse a las características de los malos y malas docentes, el aspecto psicopedagógico fue prioritariamente mencionado, tanto en el cuestionario como en la prueba de oraciones incompletas y en el conversatorio. Es rescatable, también, el señalamiento estudiantil acerca de la necesidad de que las evaluaciones de desempeño profesional, aplicadas a profesores y profesoras universitarias, tengan su impacto en el ejercicio docente, destacándose la importancia del compromiso hacia la profesión en la cual preparan y la experiencia como parte de su práctica académica.

Cabe recordar que la dimensión personal del docente y la docente involucra, entre otros aspectos, acciones asociadas a la empatía en las interacciones establecidas con sus estudiantes, y una estrecha relación entre sus ideas sobre el aprendizaje y la enseñanza, y las acciones desarrolladas en el salón de clase.

En esta línea, es esperable que exista coherencia entre el discurso del deber ser como persona y la práctica docente (Francis, 2006), aspecto que en el caso de la presente investigación, parece que fue relevante para las estudiantes participantes.

En cuanto a la categoría analítica sobre Interacciones en el aula universitaria, es interesante destacar que para las estudiantes universitarias la primera característica que define las interacciones entre las docentes y los docentes considerados como buenos y buenas y sus estudiantes, con un porcentaje suficiente ( $40 \%$ de respuestas) fueron los rasgos de personalidad referidos a : "buenas relaciones", "confianza", "solidaridad".

Al caracterizar las interacciones entre iguales, cuando los cursos están en manos de buenos y buenas docentes universitarias, las estudiantes, independientemente del énfasis de carrera, hicieron 
referencia prioritariamente, con un porcentaje suficiente (30\% de respuestas), a aspectos como los siguientes: "apoyo", "colaborativos", "compañerismo", "solidaridad”, "trabajo en grupos".

El respeto, las estrategias de trabajo, la presencia o no de relaciones de poder en los comportamientos de profesores y profesoras en el aula y la repercusión que dichos aspectos tienen en el contexto de aula, fueron reiteradamente mencionados por las estudiantes. Algunas de las expresiones textuales expresadas por las estudiantes participantes, así lo denotan: “(...) hay relaciones de poder. Hay profesores que...bueno....hay profesoras que se sienten como que ellos llegan y dan la materia y dicen esto es así y está en el libro y es así y así. Y no hay más allá de lo que ellos saben y de la experiencia que han tenido, de su conocimiento"; "”Se da inseguridad, impotencia y muchas veces, obviamente hay de todo, hay profesores y acciones de aula muy buenas pero hay otros que no. . ." (Crónicas del conversatorio).

Los datos acerca de la perspectiva estudiantil sobre el clima de aula generado por los buenos y buenas docentes universitarias, se incluyen en la siguiente figura.

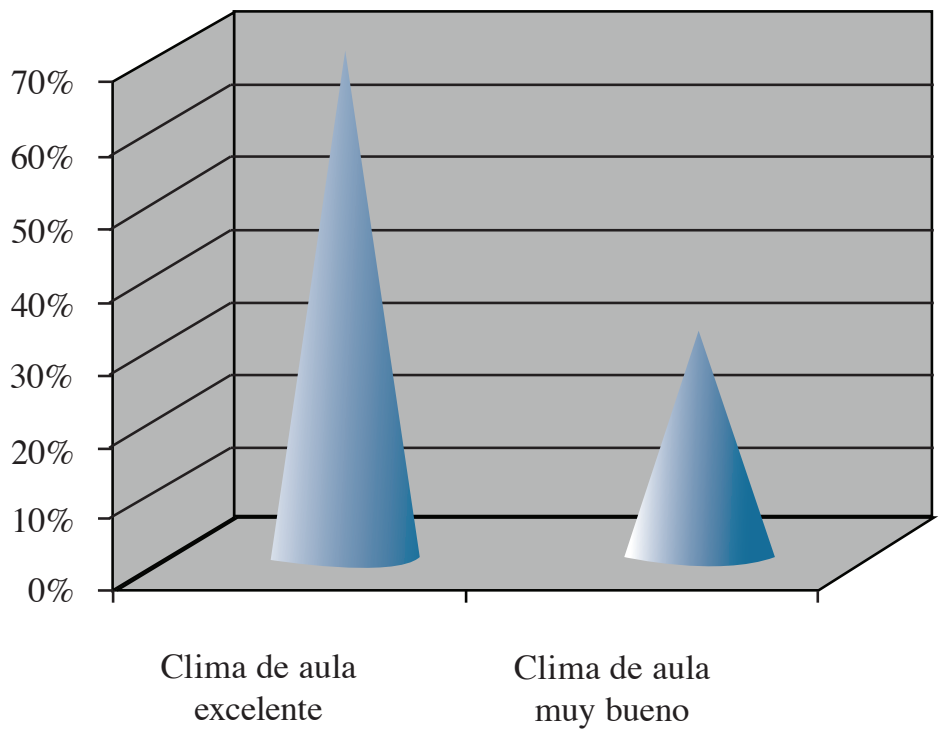

Valoración del clima de aula generado por buenos y buenas docentes

Figura 2. Valoración de las estudiantes de la UNA, acerca del clima de aula que genera un buen y buena docente, 2009.

Como se nota en los datos de la Figura 2, las estudiantes participantes, independientemente del énfasis de carrera, indicaron con un excelente porcentaje (69\% de respuestas) que el clima de aula generado cuando quien da la clase es un buen o buena docente es excelente y con un porcentaje suficiente (31\% de respuestas) lo catalogaron como muy bueno, lo que permite afirmar que para el $100 \%$ de las participantes los buenos y buenas docentes promueven climas de aula positivos para el aprendizaje. Coincide la información con los planteamientos de Allidière (2004), para quien la personalidad del docente es una variable fundamental en relación con el clima afectivo del aula. 
Es importante hacer notar que las frases expresadas por las estudiantes participantes, acerca de un buen ambiente o clima de aula, prioritariamente se refieren al respeto. En ese sentido, existe una alta coincidencia con lo aportado por Stronge (2002) (Citado por Francis, 2006) quien reporta que los valores de respeto y justicia son expresados por las estudiantes al referirse a las características de docentes excelentes.

Los datos aportados en la investigación de Pereira (2010) refieren a rasgos y características de los buenos y buenas docentes, que generan un clima de aula que bien puede denominarse como democrático, donde se dan una serie de condiciones básicas, que a criterio de las estudiantes participantes, promueven el aprendizaje. Entre lo mencionado, se destacó especialmente: que el docente y la docente universitaria se debe caracterizar por ser una persona humana, respetuosa, con adecuada comunicación, que respete las necesidades y características del estudiantado. Plantearon, además, aspectos referidos a relaciones simétricas con el estudiantado, donde se permita la participación, la expresión de ideas, donde quien ejerce la docencia muestre un buen manejo de los contenidos a enseñar. En fin, condiciones que en la bibliografía también se señalan como aspectos importantes en la práctica docente.

Las estudiantes enunciaron, del mismo modo, elementos sobre los malos y malas docentes que conllevan a la conformación de un clima de aula negativo y poco propicio para el aprendizaje. Es interesante notar, que nuevamente mencionaron entre otros aspectos: la falta de respeto, de humanismo, de comunicación, las relaciones de poder, la inflexibilidad, la falta de confianza, el manejo inadecuado de los contenidos, inadecuadas interacciones con sus alumnos y alumnas, entre lo más relevante.

Cabe resaltar que el clima de aula emerge no solo de las interacciones, del manejo de contenidos, de la estructura del aula, de las estrategias de trabajo, sino que en el mismo tiene una función primordial el papel que desempeñe el docente, este rol se constituye en el motor que impulsa una dinámica de clase que puede favorecer o no, las condiciones para un aprendizaje significativo.

Los resultados encontrados en cuanto a la dinámicas interactivas y el clima de aula, características de las buenas y buenos docentes, coincidieron plenamente con los planteamientos teóricos que señalan la importancia de que el proceso educativo universitario trascienda la institución e incorpore el aprender a vivir con los demás como uno de los pilares de la educación universitaria (Prieto, 2007).

De igual manera se plantea que ya no solo lo profesional se hace necesario, sino que, asimismo, el desarrollo de capacidades y habilidades personales y psicopedagógicas es indispensable en la práctica de la docencia universitaria (Francis, 2006).

Fueron claras las estudiantes al señalar que los rasgos positivos de los docentes y las docentes, en el ámbito personal, psicopedagógico y profesional, promueven la conformación de una clima de aula positivo, que bien puede catalogarse como democrático, es decir, generador de un ambiente de confianza, agradable y estimulador para el aprendizaje. Como condiciones básicas mencionaron, entre otras, que se requiere de docentes que sean: "humanos", "respetuosos", "favorecedores de las relaciones interpersonales", "flexibles, amigables, dan confianza, buen humor, abiertos, excelente comunicación", "muestran carisma", "explican bien la lección", "motivan al estudiantado", entre otras condiciones.

Fueron también precisas al señalar, que los docentes y las docentes que muestran rasgos negativos en áreas psicopedagógicas, personales y profesionales, a su vez promueven la conformación de climas de aula negativos y poco propicios para el aprendizaje, donde imperan las relaciones de poder, la falta de respeto, de comunicación, tales como otros aspectos señalados en los apartados precedentes. 
Es interesante indicar que la mayoría de las respuestas enunciadas como los rasgos más importantes que ha de tener un buen y buena docente universitaria, correspondieron con un muy buen porcentaje (50\% de respuestas), a aspectos propios de características personales, dentro de las cuales destacan las siguientes: "Humano", "Flexible”, "Creativo", "Empático", "Respetuoso", "Tener carisma”, "Comprensivo", "Asertividad”. Seguidos en orden decreciente, con un porcentaje suficiente (30\% de respuestas), por rasgos correspondientes a características psicopedagógicas y con un bajo porcentaje (20\% de respuestas), por rasgos profesionales.

Dado que los hallazgos indicaron una tendencia por parte de las estudiantes, independientemente del énfasis de carrera, a priorizar los rasgos de docentes, correspondientes a característica personales, se insertan a continuación los rasgos que mayoritariamente fueron mencionados por ellas como necesarios de ser encontrados en cualquier docente universitario.

\begin{tabular}{|l|l|l|l|}
\hline "Respeto" & "Humanidad" & "Empatía" & "Tolerancia" \\
\hline "Amigable" & "Amistad" & "Humilde" & "Carisma" \\
\hline "Ser buena persona" & "Afectivo" & "Expresivo" & "Entusiasta" \\
\hline $\begin{array}{l}\text { "Establecer buenas relacio- } \\
\text { nes" }\end{array}$ & "Que sonría" & "Sinceridad" & "Comprensión" \\
\hline "Motivación" & "Comunicativo" & "Apertura" & "Buen humor" \\
\hline "Flexibilidad" & "Ordenado" & "Asertividad" & $\begin{array}{l}\text { "Confianza" } \\
\text { "Seguro" }\end{array}$ \\
\hline "Disposición" & "Propositivo" & "Emprendedor" & "Solidaridad" \\
\hline
\end{tabular}

Es interesante destacar cómo el respeto y los rasgos que demuestran mayor humanidad son reiteradamente señalados por las estudiantes. Me parece muy significativo, por ejemplo, que el buen humor, la sonrisa, la afectividad, en general con señalamientos como: amigable, expresivo, asertivo, empático, fueron valorados por las estudiantes. En ese sentido, hay coincidencia con los planteamientos teóricos más recientes, mencionados en párrafos anteriores, los cuales proponen que las emociones han de ser parte de los procesos de enseñanza y aprendizaje, y sugieren la necesidad de retomar condiciones de aula que prioricen la incorporación del afecto como elemento importante para el aprendizaje significativo.

\section{Discusión de resultados}

Existe coincidencia entre los planteamientos teóricos acerca de las características de los docentes y las docentes universitarias (profesionales, psicopedagógicas y personales) y las respuestas que dan las estudiantes participantes en la investigación.

Los datos mostraron como aspectos relevantes para las estudiantes, las características personales, psicopedagógicas y profesionales de los docentes y las docentes universitarias, identificándolas como determinantes en la conformación de condiciones favorables o no, para los procesos de enseñanza y aprendizaje. Estas mismas categorías se encuentran mencionadas en labibliografía como globalizadoras de rasgos importantes en el grupo de docentes. No obstante lo dicho, es más 
frecuente encontrar referencias teóricas acerca de sus características profesionales y su importancia, no así acerca de las características personales, aspecto que en los resultados de la investigación fueron relevantes.

Mata (2004) señala que el profesionalismo, entendido como la formación en una área de especialidad, es la condición de buen maestro a la cual se le suele otorgar mayor importancia en el nivel social; sin embargo, en el caso concreto de la investigación a que hace referencia el presente artículo, esa categoría es la tercera en orden de importancia para las estudiantes participantes, pues estas priorizan las características personales y, en segundo lugar, las psicopedagógicas.

No obstante, las características profesionales del cuerpo docente fueron mencionadas reiteradamente en los tres niveles de importancia para los cuales se pidieron características de los y las docentes.

Al profundizar sobre las razones para otorgar un primer lugar de importancia a las características personales de los docentes y las docentes, las estudiantes fueron claras al señalar, que los docentes y las docentes universitarias en carreras de formación pedagógica se constituyen en modelos que serán imitados a futuro cuando las estudiantes estén en su práctica profesional como docentes. Es esa la razón primordial por la cual ellas argumentaron que el grupo de docentes universitarios han de preocuparse por el trato que dan a sus estudiantes, e incluso manifestaron que en otras carreras podría no ser tan relevante el hecho de que un docente no trate bien a sus alumnos, pero que esto es inaceptable en carreras de pedagogía.

Desde el 2008, gracias al planteamiento de un profesor de la carrera de Química de la Universidad Nacional, se formuló una denuncia pública en el foro universitario, que el autor denominó "terrorismo académico", en ese sentido se preguntaba quiénes fallaban, si el estudiantado o el profesorado. En su momento argumentó que en algunas aulas universitarias el grupo estudiantil recibía “. . . un trato hostil basado en gritos, amenazas e insultos” (Sánchez, 2008).

Más recientemente, en la Universidad Nacional, se ha generado una discusión académica vía internet, en la cual se han hecho fuertes señalamientos acerca de docentes universitarios que infunden temor en sus clases y que generan un clima de desconfianza, falta de armonía, donde las relaciones de poder por su parte son evidentes (Sánchez (2009); Méndez (2009); Morales (2009); Piedra (2009); Rodríguez (2009), entre otros).

Dichos señalamientos parecieran sugerir que, independientemente de la carrera, es importante, para el ejercicio docente universitario, el procurar establecer interacciones armoniosas en el salón de clase, donde se busque la conformación de un clima que propicie el aprendizaje, el compartir, la expresión de ideas sin temor, la divergencia de planteamientos y, en general, un sano crecimiento intelectual y personal, no solo para los grupos estudisntiles, sino también para el mismo profesorado universitario. Y con esos planteamientos cobran mayor relevancia los señalamientos estudiantiles, que aporta la presente investigación, pues los mismos tienen una fuerte coincidencia con los argumentos emitidos en el foro universitario.

Por otra parte, Caputo (2007) plantea que la innovación educativa ha de trascender los métodos, técnicas y programas y acercarse a la incorporación, reconocimiento y análisis de aspectos de carácter afectivo que podrían incidir en los procesos de aprendizaje. En este sentido, los datos encontrados mostraron que las estudiantes participantes, tanto mediante el Cuestionario, como en las Oraciones Incompletas y el Conversatorio, son consistentes en señalar la importancia de contar con docentes humanos, afectivos, comprensivos, características que, entre otras, fueron destacadas como relevantes para el ejercicio docente.

Es interesante señalar que, aunque hay una clara priorización de las características personales positivas del profesor y la profesora, los estudiantes no dejaron de lado aspectos como la entrega de 
la docencia y el dominio de las temáticas impartidas. Para las estudiantes participantes, es necesario, además, que estén presentes características que les permitan a los docentes un buen trabajo con el manejo de los contenidos y las estrategias de aprendizaje en el trabajo de aula.

En el sentido señalado, los planteamientos de las estudiantes, retoman características docentes que se encaminan hacia la formación integral, donde se destacan no solo aspectos cognoscitivos, sino también valores, comportamientos éticos y un claro interés por ambientes de sana convivencia. Aspectos que tienen total concordancia con los señalamientos de autores como Prieto (2007); Francis (2006); Palomero y Fernández (1999); Ortiz y Mariño (2005); Campos (2003) y Pereira (2008-b), entre otros.

La diferencia encontrada con los planteamientos teóricos estriba en el hecho de que los autores y autoras suelen indicar que lo profesional es lo más importante en la entrega de la docencia. No obstante, desde las vivencias y anhelos de las estudiantes participantes, al expresar características deseables que debe tener todo docente universitario, se encontró que estas priorizaron los rasgos personales sobre los psicopedagógicos y los profesionales.

Lo mencionado es importante de considerar, dado que también las revisiones teóricas realizadas (Hernández (2005); Francis (2006); Depraz (2005); Ibáñez, Barrientos, Delgado, Figueroa y Geisse (2004), entre otros) señalan con claridad que los aspectos afectivos y motivacionales, así como las emociones y los sentimientos, permean todo el proceso de aprendizaje.

En concordancia con lo anterior, parece lógico suponer que en investigaciones previas, donde no han mediado necesariamente las perspectivas estudiantiles, podría haber existido una predominancia de lo profesional sobre lo personal, aspecto que en esta investigación se está invirtiendo, dado que la tendencia observada fue la primacía de lo personal, pero sin descuidarse por parte de las estudiantes, las características psicopedagógicas y profesionales de docentes universitarios.

Se demostró, asimismo, que para las estudiantes participantes, es importante la presencia de rasgos positivos de personalidad. Manifestaron que especialmente en carreras de Educación, donde los modelos docentes se constituyen también en elementos de aprendizaje y duplicación cuando ellas estén con sus propios alumnos, es una posible razón para que hayan priorizado las características de personalidad en los docentes y las docentes.

Además es posible suponer que podría existir una idea en el estudiantado, ligada a la conceptualización de buenos y buenas docentes, en el sentido de asumir que quien es considerado como buen o buena docente, necesariamente ha de demostrar un nivel profesional alto y habilidades psicopedagógicas también altas, lo cual, entonces, les hace llevar a decir que si tiene esas habilidades, lo más importante es que resalte en los aspectos personales.

Las estudiantes anhelan docentes caracterizados por su humanismo, solidaridad, comprensión, alegría, entusiasmo, entre otros aspectos. De manera muy reiterada, señalan la necesidad de que el respeto esté presente en las dinámicas interactivas en el aula universitaria.

Hay un llamamiento de las estudiantes para que sus profesores y profesoras sean más creativos y menos tradicionales; que las clases no sean magistrales ni aburridas; que expliquen bien los contenidos, y sean ordenados y ordenadas entre otros, aspectos; todos referidos a sus capacidades profesionales y psicopedagógicas. Coinciden los datos tanto del Cuestionario como de las Oraciones Incompletas, en cuanto a la importancia de características de personalidad, psicopedagógicas y profesionales.

Es clara la perspectiva estudiantil acerca de las características de los docentes y las docentes universitarias y el clima de aula, cuando, de manera mayoritaria, argumentan que los rasgos personales son prioritarios en docentes que realizan su ejercicio profesional en la formación de carreras en Pedagogía. 
Igualmente, hubo un señalamiento de las estudiantes participantes, acerca de la importancia que debieran tener los procesos de evaluación del desempeño docente que se aplican en la Universidad. Desde su perspectiva, se manifestaron insatisfechas con los mismos, pues argumentan que los malos docentes y las malas docentes lo siguen siendo a lo largo de los años, pues aunque mediante esas evaluaciones se denuncian aspectos que generan descontento en el estudiantado, la Universidad no realiza el seguimiento ni propone estrategias para el mejoramiento de dichos académicos. En esta línea manifestaron que es urgente la necesidad de que la institución contrate a docentes que demuestren excelencia mediante las evaluaciones del desempeño y no se nombre a quienes son mal evaluados por el estudiantado.

De igual manera, plantearon la importancia de que los rasgos de personalidad estén en concordancia con la formación profesional y con habilidades psicopedagógicas. Destacaron, en ese sentido, el papel tan predominante que tienen los docentes y las docentes en la conformación de un clima de aula favorable o no para el aprendizaje. En este sentido, queda claro, también, que las dinámicas interactivas en el aula universitaria van más allá que la mera relación entre docentes y alumnas. Se relacionan también con ellas, los contenidos del currículo, las estrategias y recursos didácticos que tenga la habilidad de poner en práctica el profesorado, las relaciones de poder, las emociones, y las dinámicas entre iguales; aspectos todos que reiteradamente han señalado. Igualmente ha quedado clara la importancia que las estudiantes de licenciatura, independientemente del énfasis de carrera, otorgaron a los rasgos correspondientes a características de personalidad de docentes y su repercusión en la conformación del clima de aula favorable, democrático y propicio para un aprendizaje integral.

La triangulación realizada entre teoría, informantes e instrumentos, marcó fuertemente las tendencias arriba señaladas, permitiendo mediante el método fenomenológico la profundización en la temática y la ratificación de coincidencias mediante el proceso de triangulación.

\section{Conclusiones}

Se enumeran a continuación las conclusiones más relevantes aportadas por la investigación:

- $\quad$ Son pocos los estudios universitarios en los cuales se retoma la opinión estudiantil en aspectos referidos a los docentes y las docentes. Excepto en aquello que tiene que ver con la valoración de algunos aspectos pedagógicos como parte de la evaluación institucional o de procesos de acreditación de carreras.

- $\quad$ La utilización de un diseño de método mixto fue valioso para el abordaje de la temática sobre caracterización de docentes y el clima de aula, desde la perspectiva estudiantil.

- $\quad$ Es novedoso el aporte de la investigación en el sentido de ofrecer una mirada estudiantil acerca del grupo universitario de docentes, dado que la mayoría de las investigaciones no incorporan al estudiantado como fuente primaria de información.

- $\quad$ Existe diferencia evidente entre los planteamientos teóricos, que destacan las características profesionales de docentes y los planteamientos de las estudiantes, que reiteradamente priorizan las características personales, seguidas de las psicopedagógicas y las profesionales.

- Se identificó la tendencia estudiantil, independientemente del énfasis de carrera, a señalar como prioritarios, los rasgos de características personales de sus docentes universitarios, por lo que, de manera generalizada, dicha categoría emerge.

- $\quad$ El respeto es reiteradamente señalado por las estudiantes como un factor decisivo en la calidad de las dinámicas interactivas, las características de los buenos y buenas docentes 
universitarias y el adecuado clima de aula, coincidiendo con lo planteado por Stronge, 2002 (Citado por Francis, 2006), cuando plantea que los valores de respeto y justicia son señalados por los estudiantes cuando se refieren a las características de docentes excelentes.

- $\quad$ Desde la perspectiva estudiantil hay una relación estrecha entre el tipo de profesor o profesora y el clima de aula que se genera en las aulas universitarias.

- $\quad$ La totalidad de las estudiantes participantes, independientemente del énfasis de la carrera que cursaban, indicaron que el clima de aula que se genera cuando las clases las da un buen docente o una buena docente universitaria, es excelente y muy bueno.

- $\quad$ La totalidad de las estudiantes, independientemente del énfasis de carrera, definieron que el clima de aula, de cursos a cargo de un mal docente o una mala docente universitaria, es muy malo, malo y regular.

- La mirada estudiantil señaló, claramente, que en los cursos a cargo de "malos" y "malas" docentes, se identifican relaciones de poder inherentes a las interacciones entre el cuerpo docente y sus estudiantes, caracterizadas por la incapacidad de enfrentarse a ellas, por parte de la mayoría del estudiantado. De igual manera destacaron que, en dichos cursos, se evidencia un mal manejo del contenido por parte de sus docentes, por lo que detallaron una serie de aspectos referidos a las características psicopedagógicas y profesionales de dichos docentes.

- Este aspecto coincide con algunos de los planteamientos teóricos de Parra (1992), Escofet (Citado por Rivera, s. f); Chaves (2006); Pereira (2008-a, 2008-b); Naranjo (2007), entre otros, quienes apuntan que en la actualidad las relaciones de poder en el aula universitaria son parte de la cotidianidad.

- $\quad$ Aunque las estudiantes priorizaron de manera enfática los rasgos personales de sus docentes como característica que debe tener en primer lugar, cualquier buen o buena docente en la universidad, es válido indicar, que los principales rasgos por ellas señaladas, en orden de importancia, son propios de las siguientes características: p de manera generalizada de manera generalizada de manera generalizada ersonales, psicopedagógicas y profesionales.

\section{Referencias bibliográficas}

Allidière, N. (2004). El vínculo profesor-alumno. Una lectura psicológica. Buenos Aires: Editorial Biblos.

Camacho, S. y Mendías, A. M. (s. f.) Aportación a la mejora de la calidad en la formación de maestros. OEI Revista Iberoamericana de Educación, 1-17. Disponible en http://www.rieoei.org/ deloslectores/900Camacho.PDF

Campos, N. (2003). El docente investigador: su génesis teórica y sus rasgos. Revista Educación (27)2, 39-43.

Caputo, M. (Setiembre-Diciembre, 2007). O enigma da inclusão: das intenções às práticas pedagógicas. [El enigma de la inclusión: de las intenciones a las prácticas pedagógicas]. Educação e Pesquisa 33(3), 543-560. Recuperado el 18 Abril, 2008 de http://www.scielo.br/scielo. php?script=sci arttext\&pid=S15179702200700030011\&lng=en\&nrm=iso 
Chaves, A. L. (2006). La construcción de subjetividades en el contexto escolar. Revista Educación 30(1), 187-200.

Cresswell, J. (2003). Outline: Creswell's Research Design. Recuperado el 27 Mayo, 2009 de http:// www.ics.uci.edu/alspaugh/human/crewel.html

Dellinger, A. y Leech, N. (2007). Toward a Unified Validation Framework in Mixed Methods Research. [Hacia un marco unificado de validación de métodos de investigación mixtos]. Journal of Mixed Methods Research, 1(4), 309-332. Recuperado el 12 Agosto, 2009 de http:// mmr.sagepub.com/cgi/content/abstract/1/4/309

Depraz, N. (Julio-Diciembre, 2005). A ética relacional: uma prática de ressonância interpessoal. [La ética relacional: uma práctica de resonancia interpersonal]. Revista do Departamento de Psicologia-UFF, 17(2), 19-34. Recuperado el 09 Marzo, 2007 de http://www.scielo.br/scielo. php?script=sci arttext\&pid=S0104-80232005000200003\&lng=en\&nrm=iso

Di Silvestre, C. (2008). Metodología Cuantitativa versus Metodología Cualitativa y los Diseños de Investigación Mixtos: Conceptos Fundamentales. Argentina: Universidad de los Andes. ANACEM. Recuperado el 07 Julio, 2009 de http://www.anacem.cl/wp-content/ uploads/ 2008/07/ metodologia-cuantitativa-versus- cualitativa.pdf

Driessnack, M., Sousa, V. y Costa, I. (Setembro, outubro, 2007). Revisão dos Desenhos de Pesquisa Relevantes para Enfermagem: parte 3: Métodos Mistos e Múltiplos. [Revisión de diseños relevantes para enfermería: métodos mixtos y múltiples: Parte 3]. Rev Latino-am Enfermagem 15(5), 179-182. Recuperado el 24 Enero, 2008 de http://www.scielo.br/pdf/rlae/v15n5/pt v15n5a24.pdf

Francis, S. (2006). Hacia una caracterización del docente universitario "excelente": una revisión a los aportes de la investigación sobre el desempeño docente. Revista Educación 30(1), 31-49.

Gallardo, L. (2007). Las estrategias comunicativas de alumno y profesor en el aula. Recuperado el 14 diciembre, 2009 de http://www.aprendemas.com/Noticias/html/N1954_F15012007.HTM

Hernández, A. (2005). Los factores afectivos y el aprendizaje significativo en la docencia universitaria. Tesis de maestría sin publicar. Universidad Nacional. Heredia, Costa Rica.

Hernández, R., Fernández, C. y Baptista, P. (2004). Metodología de la investigación (3ª ed.). México: Editorial Mc Graw-Hill.

Ibáñez, N., Barrientos, F., Delgado, T., Figueroa, A., Geisse, G. (2004). Las Emociones en el Aula: Percepción de los académicos. Avances de Investigación, $\mathrm{N}^{\circ}$ 2, 1-10. Recuperado el 05 Noviembre, $2007 \mathrm{de}$ http://www.umce.cl/ investi/avance n ibanez.html

Johnson, B. y Onwuegbuzie, A. (2004). Mixed methods Research: A Research Paradigm Whose Time Has Come. [Métodos mixtos de investigación: Un paradigma de investigación cuyo 
tiempo ha llegado]. Educational Researcher, 33(7), 14-26. Recuperado el 02 Febrero, 2008 de http://edr.sagepub.com/cgi/content/abstract/33/7/14

Laborda, C. y Essomba, M. (Junio, 2000). Aprendizaje activo sobre "Atención a la Diversidad” en los estudios de magisterio: una propuesta de programa. Barcelona: Congreso Internacional de Docencia Universitaria e Innovación. Recuperado el 26 Abril, 2009 de http://webs2002. uab.es/paplicada/htm/papers/Paper_aprendizajeactivo_essomba.htm

Mata, A. (2004). El sentido social de la idea de un buen maestro. Revista Electrónica Actualidades Investigativas en Educación 4(2), 1-38. Consultado el 26 Marzo, 2008 de http://revista.iimec. ucr.ac.cr/articulos/2-2004/archivos/maestro.pdf

Méndez, C. (2009, 23 setiembre). Fascinación Académica vrs Miedo en las Aulas. [Foro desarrollado por correo electrónico]. Consultado el 23 Setiembre, 2009 de http://www.una.ac.cr

Morales, G. (2009, 21 setiembre). Los Verdaderos Maestros. [Foro desarrollado por correo electrónico]. Consultado el 21 Setiembre, 2009 de http://www.una.ac.cr

Naranjo, M. L. (2007). Autoestima: un factor relevante en la vida de la persona y tema esencial del proceso educativo. En: Revista Electrónica Actualidades Investigativas en Educación 7(3), 1-27 Consultado el 04 Mayo, 2008 de http://revista.inie.ucr.ac.cr/articulos/3-2007/archivos/ autoestima.pdf

Ortiz, E. y Mariño, M. (2005) La profesionalización del docente universitario a través de la investigación didáctica desde un enfoque interdisciplinar con la psicología. Cuba: Universidad de Holguín. Revista Iberoamericana de Educación, ISSN: 1681-5653. Disponible en: www. rieoei.org/deloslectores/8880rtiz.PDF.

Palomero, J. y Fernández, M. (1999). Enseñando y aprendiendo en la Universidad. Revista Electrónica Interuniversitaria de Formación del Profesorado 2(1), 369-399. Consultado el 13 Octubre, 2008 de Disponible en: www.uva.es/aufop/publica/revelfop/99-v2n1/htm

Parra, R., González, A., Moritz, O., Blandon, A. y Bustamante, R. (1992). La escuela violenta. Colombia: Tercer mundo editores.

Pereira, Z. (2010). La mirada de estudiantes de la Universidad Nacional hacia el docente y la docente: sus características y clima de aula. Tesis de doctorado sin publicar. Universidad Estatal a Distancia. San José, Costa Rica.

Pereira, Z. (2008-a). Las relaciones de poder en el aula universitaria (1 ${ }^{\mathrm{a}}$ parte). Revista Visión Docente Con-Ciencia. Año VII. No. 40, 24-31.

Pereira, Z. (2008-b). Las relaciones de poder en el aula universitaria (2a parte). Revista Visión Docente Con-Ciencia. Año VII. No. 41, 23-31. 
Piedra, G. (2009, 21 setiembre). Terrorismo Académico y Demás. [Foro desarrollado por correo electrónico]. Consultado el 21 Diciembre, 2009 de http://www.una.ac.cr

Polanco, A. (2005). La motivación en los estudiantes universitarios. Revista Electrónica Actualidades Investigativas en Educación 5(2), 1-13. Consultado el 07 Enero, 2008 de http://revista.inie.ucr. ac.cr/articulos/2-2005/archivos/motivacion.pdf

Prieto, A. T. (mayo-agosto, 2007). El profesorado universitario: su formación como modelo de actuación ética para la vida en convivencia. Revista Electrónica. Actualidad Investigativas en Educación. 7(2), 1-20. Consultado el 23 Noviembre, 2008 de http://revista.inie.ucr.ac.cr

Rivera, Y. (s. f.). La cotidianidad de un salón de clases: un espacio de vivencias para comprender la evaluación. San José, Costa Rica: Universidad Estatal a Distancia.

Rocco, T., Bliss, L, Gallagher, S. y Pérez, A. (2003). Taking the next step: mixed methods research in organizacional systems. [Tomando el siguiente paso: los métodos de investigación mixtos en los sistemas organizacionales]. Information Technology, Learning and Performance Journal, 21(1), 19-29.

Rodríguez, G., Gómez, J. y Gil, J. (1996). Métodos de la Investigación Cualitativa. Málaga: Ediciones ALJIBE.

Rodríguez, M. (2009, 18 setiembre). Pedagogía del Miedo. [Foro desarrollado por correo electrónico]. Consultado el 18 Setiembre, 2009 de http://www.una.ac.cr

Salgado, A. (setiembre, 2007). Investigación cualitativa: diseños, evaluación del rigor metodológico y retos. Liberabit, 13(13), 71-78. Disponible en http://www.scielo.org.pe/pdf/liber/v13n13/ a09v13n13.pdf

Sánchez, R. (2008, 24 noviembre). ¿Quién falla: el profesor o el estudiante? [Foro desarrollado por correo electrónico]. Consultado el 22 Noviembre, 2008 de http://www.una.ac.cr

Sánchez, R. (2009, 23 setiembre). Enseñar es un Arte. [Foro desarrollado por correo electrónico]. Consultado el 23 Setiembre, 2009 de http://www.una.ac.cr

Valles, M. (1999). Técnicas cualitativas de investigación social. Reflexión metodológica y práctica profesional. Vallehermoso, Madrid: Editorial Síntesis, S. A. 